Noise analysis of geometrically complex mechanical structures using the analogy between electrical circuits and mechanical systems

G. G. Yaralioglu, and A. Atalar

Citation: Review of Scientific Instruments 70, 2379 (1999);

View online: https://doi.org/10.1063/1.1149766

View Table of Contents: http://aip.scitation.org/toc/rsi/70/5

Published by the American Institute of Physics

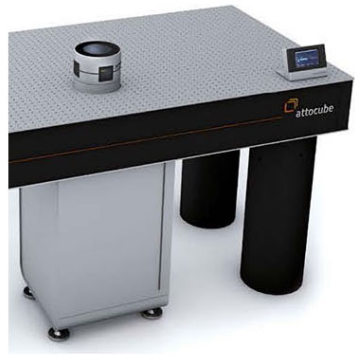

Obstruction free access
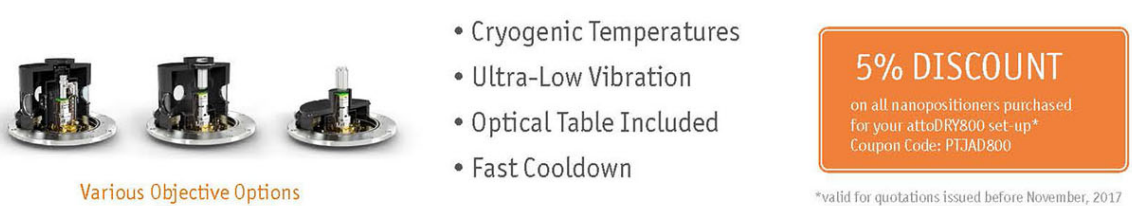


\title{
Noise analysis of geometrically complex mechanical structures using the analogy between electrical circuits and mechanical systems
}

\author{
G. G. Yaralioglua) and A. Atalar \\ Electrical and Electronics Engineering Department, Bilkent University, Ankara, Turkey 06533
}

(Received 21 August 1998; accepted for publication 5 February 1999)

\begin{abstract}
Random fluctuations of displacement or velocity in mechanical systems can be calculated by using the analogy between electrical circuits and mechanical systems. The fluctuation-dissipation theorem expresses the relation between the generalized mechanical admittance and the noise in velocity. Similarly, correlation of mechanical noise can be calculated by using the generalized Nyquist theorem which states that the current noise correlation between two ports in an electrical circuit is dictated by the real part of the transadmittance. In this article, we will present the determination of the mechanical transadmittance and we will use the mechanical transadmittance to calculate the noise correlation on geometrically complex structures where it is not possible to approximate the noise by using the simple harmonic oscillator model. We will apply our method to atomic force microscope cantilevers by means of finite element method tools. The application of the noise correlation calculation method to rectangular cantilever beams shows some interesting results. We found that on the resonance frequencies, the correlation coefficient takes values 1 (full correlation) and -1 (anti-correlation) along the cantilever axis depending on the mode shapes of the structure.

(C) 1999 American Institute of Physics. [S0034-6748(99)04005-8]
\end{abstract}

\section{INTRODUCTION}

Random vibrations of mechanical parts set the fundamental limit on the minimal detectable displacement in many precision displacement measuring devices like the atomic force microscope (AFM), laser interferometric gravitational wave detectors, accelerometers, etc. Similar to the series voltage noise of a resistor in an electrical circuit, the source of random vibrations is the thermal agitation. To determine the merit of a displacement sensing system, it is crucial to understand this noise source and calculate the amplitude of the random vibrations.

The resolution of an AFM system which uses an optical detection method is determined by the thermal noise of the cantilever. In an optical AFM system, the cantilever is illuminated by a laser beam and the reflected beam from the cantilever is detected by a photodetector. Since the laser integrates the mechanical noise within its beam, the correlation of noise plays an important role in the output noise.

Thermal noise of an AFM cantilever has been intensively studied. ${ }^{1-5}$ In most of the earlier work, the cantilever has been modeled by a simple harmonic oscillator and the noise of the free end has been calculated by employing the equipartition theorem. ${ }^{6}$ However, the output noise calculation of the optical AFM system using the simple harmonic oscillator model assumes that the noise within the laser illumination is fully correlated. As we will show in Sec. IV, this is a good approximation to predict the output noise for simple AFM cantilevers since the noise correlation varies slowly at the free end. However, for geometrically complex structures like interdigital cantilevers ${ }^{7,8}$ which are composed

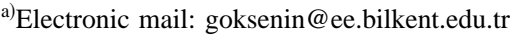

of two sets of interleaving fingers, the noise correlation between fingers should be calculated for an accurate analysis of the noise performance.

In this work, we will present a method for the calculation of noise and noise correlation for geometrically complex mechanical structures. The structure may be made up of different materials or may have different loss coefficients in different parts of the structure. For the noise calculation, we will employ the analogy between electrical circuits and mechanical systems. First, electrical noise equations will be presented. We will repeat the result of the fluctuationdissipation theorem ${ }^{9,10}$ for the calculation of noise at a single point, then present the calculation of noise correlation between two points on the mechanical structure. For the mechanical response calculations, we will use the finite element method (FEM). Depending on the FEM meshing of the mechanical structure, we will define the noise correlation matrix corresponding to the mechanical structure.

We will also define the noise correlation coefficient which can be used to estimate the degree of noise correlation between different parts of the structure.

\section{ELECTRICAL NOISE EQUATIONS}

In dissipative linear electrical systems, thermal or Johnson noise was characterized by Nyquist ${ }^{11}$ in 1928 . The thermal noise of a two terminal network can be represented by a series voltage noise source, $e$, or by a shunt current noise source, $i$, as determined by the Nyquist theorem. The mean values of the source phasors are zero, $\langle e\rangle=0,\langle i\rangle=0$, and the mean square noise voltage or noise current is determined by the real part of the impedance $(Z)$ or the admittance $(Y)$ seen between the terminals 


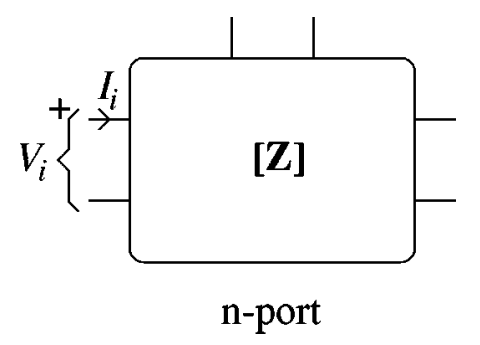

FIG. 1. General $n$-port network.

$$
\begin{gathered}
\left\langle e^{2}\right\rangle=4 k_{B} T \Delta f \mathcal{R} \operatorname{e}\{Z\}, \\
\left\langle i^{2}\right\rangle=4 k_{B} T \Delta f \mathcal{R} \operatorname{Re}\{Y\},
\end{gathered}
$$

where $e$ and $i$ is the root mean square (rms) noise voltage and noise current, respectively, and $\mathcal{R} e\{\}$ denotes the real part; $k_{B}$ is the Boltzmann constant and $T$ is temperature in Kelvin. Infinitesimal measurement bandwidth is denoted by $\Delta f$. In general, $Z$ or $Y$ is a function of frequency and the above equations are valid in the narrow frequency band of $\Delta f$.

The Nyquist theorem can be generalized to multiterminal networks to include the correlation of noise between the two ports. ${ }^{12}$ Consider a general dissipative linear passive $n$-port network (Fig. 1) with a Z-parameter matrix

$$
\underbrace{\left[\begin{array}{c}
V_{1} \\
V_{2} \\
\cdot \\
V_{n}
\end{array}\right]}_{\mathbf{V}}=\underbrace{\left[\begin{array}{ccccc}
Z_{11} & Z_{12} & \cdot & \cdot & Z_{1 n} \\
Z_{21} & Z_{22} & \cdot & \cdot & Z_{2 n} \\
\cdot & \cdot & \cdot & \cdot & \cdot \\
Z_{n 1} & Z_{n 2} & \cdot & \cdot & Z_{n n}
\end{array}\right]}_{\mathbf{Z}} \underbrace{\left[\begin{array}{c}
I_{1} \\
I_{2} \\
\cdot \\
I_{n}
\end{array}\right]}_{\mathbf{I}},
$$

where $\mathbf{V}=\left[V_{1} V_{2} \ldots V_{n}\right]^{T}$ is the column vector of port voltages, $\mathbf{I}=\left[I_{1} I_{2} \ldots I_{n}\right]^{T}$ is the column vector of port currents and $Z$ is the impedance matrix. An equivalent representation can be given as

$$
\mathbf{I}=\mathbf{Y} \mathbf{V},
$$

where $\mathbf{Y}$ is the admittance matrix $\left(\mathbf{Y}=\mathbf{Z}^{-1}\right)$. If $e_{i}$ shows the open circuit noise voltage at port $i$, then the mean value of $e_{i}$ is zero, and the mean square noise voltage is given by

$$
\left\langle e_{i}^{2}\right\rangle=4 k_{B} T \Delta f \mathcal{R e}\left\{Z_{i i}\right\}
$$

Hence, the mean square noise voltage of the open-circuited port is determined by the real part of the open-circuit impedance at the corresponding port. Equivalently, the mean square noise current is

$$
\left\langle i_{i}^{2}\right\rangle=4 k_{B} T \Delta f \mathcal{R} \operatorname{e}\left\{Y_{i i}\right\} .
$$

Moreover, it is also possible to calculate the correlation of noise between ports. Correlation of the noise voltages between port $i$ and $k$ is given by

$$
\left\langle e_{i} e_{k}^{*}\right\rangle=4 k_{B} T \Delta f \mathcal{R} \mathrm{e}\left\{Z_{i k}\right\},
$$

where $*$ represents the conjugate operation. For reciprocal circuits, the $\mathbf{Z}$ matrix is symmetric $\left(Z_{i k}=Z_{k i}\right)$. In general, the above equations are also valid for nonreciprocal circuits. ${ }^{12}$ Equation (2.7) states that the correlation of the

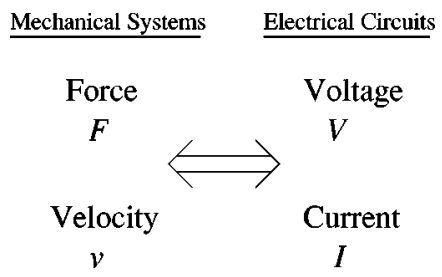

FIG. 2. Analogy between mechanical systems and electrical circuits.

noise voltages is determined by the real part of the transimpedance. Equivalently, correlation of noise currents is dictated by the real part of the transadmittance

$$
\left\langle i_{i} i_{k}^{*}\right\rangle=4 k_{B} T \Delta f \mathcal{R e}\left\{Y_{i k}\right\} \text {. }
$$

\section{NOISE IN MECHANICAL STRUCTURES}

Results obtained from the Nyquist theorem can be extended to lossy mechanical systems. ${ }^{10}$ Any linear mechanical structure can be represented as a three-dimensional electrical circuit with nodes arranged like a finite element model of the mechanical structure. ${ }^{13}$ The inductive elements represent the springs and the capacitive elements represent the mass of the mechanical structure. Resistive elements stand for the losses in the structure. The mutual inductors can be used to represent the coupling between different directions. For mechanical systems, a driving point admittance at any point may be defined and the mechanical noise at this point in the structure can be found from the real part of this driving point admittance. ${ }^{10}$ For an accurate analysis of noise performance, it is also necessary to calculate the correlation of noise between the two points on the mechanical structure. Noise correlation can be calculated by using Green's functions and damping parameters. ${ }^{14}$ For this calculation, we will use the electrical analog of this problem and the correlation of the noise at any two points will be related to the real part of the mechanical transimpedance or transadmittance.

Mechanical systems and electrical circuits are analogs of each other (Fig. 2). Mechanical equations expressing the relation between force and velocity can be obtained by replacing voltage, $V$, with force, $F$, and current, $I$, with velocity, $v$. By using this convention, we can define the driving point resistance (real part of the driving point impedance) of point $i$ on the mechanical structure as the real part of the ratio of the $F$ to $v$ :

$$
R_{i i}=\mathcal{R e}\left\{\frac{F_{i}}{v_{i}}\right\},
$$

where $F_{i}$ and $v_{i}$ are phasors. $F_{i}$ is the applied force to point $i$ and $v_{i}$ is the resulting velocity. The amplitude of the mechanical thermal noise force acting on this resistance is given by

$$
\left\langle F_{n_{i}}^{2}\right\rangle=4 k_{B} T \Delta f R_{i i} .
$$

Similarly, the mechanical driving point conductance (real part of the driving point admittance) is defined as

$$
G_{i i}=\mathcal{R e}\left\{\frac{v_{i}}{F_{i}}\right\}
$$


and the mechanical noise in velocity is

$$
\left\langle v_{n_{i}}^{2}\right\rangle=4 k_{B} T \Delta f G_{i i},
$$

where $\left\langle v_{n_{i}}^{2}\right\rangle$ shows the mean square mechanical noise amplitude in the velocity of point $i$ on the mechanical structure.

The determination of the driving point admittance involves applying a sinusoidal force to a specific point $i$ and measuring the velocity of the same point. If there is no loss in the mechanical structure, the phase difference between the force phasor and the velocity phasor will be $90^{\circ}$ or $-90^{\circ}$. Hence, Eqs. (3.2) and (3.4) will give zero noise. Similarly, we can find the transadmittance between two points by applying a sinusoidal force to the point $i$ and measuring the velocity of the other point $k$. Transconductance is given by

$$
G_{i k}=\mathcal{R e}\left\{\frac{v_{k}}{F_{i}}\right\} .
$$

By using the mechanical analog of Eq. (2.8), the correlation of the velocity noise between two points is given by

$$
\left\langle v_{n_{i}} v_{n_{k}}^{*}\right\rangle=4 k_{B} T \Delta f G_{i k} .
$$

It is customary to use the rms displacement noise, rather than the noise in velocity. We can easily derive noise equations for displacement, $u$, from Eqs. (3.4) and (3.6). Since velocity is the derivative of displacement, the Nyquist relation for the displacement noise is

$$
\left\langle u_{n_{i}}^{2}\right\rangle=4 k_{B} T \Delta f \operatorname{Im}\left\{-\frac{1}{\omega} \frac{u_{i}}{F_{i}}\right\}
$$

and the correlation of displacement noise between point $i$ and $k$ is

$$
\left\langle u_{n_{i}} u_{n_{k}}^{*}\right\rangle=4 k_{B} T \Delta f \operatorname{Im}\left\{-\frac{1}{\omega} \frac{u_{k}}{F_{i}}\right\},
$$

where $\operatorname{Im}\{\}$ denotes the imaginary part and $\omega=2 \pi f$ is the radial measurement frequency. For displacement we can define a noise correlation matrix, $\mathbf{N}$, where the diagonal elements show the mean square absolute displacement noise at point $i$ and the off-diagonal elements are the correlation of displacement noise between points $i$ and $k$ :

$$
N_{i k}=\left\langle u_{n_{i}} u_{n_{k}}^{*}\right\rangle .
$$

The noise correlation matrix is symmetric for reciprocal systems.

\section{APPLICATION OF THE METHOD: CANTILEVER BEAM}

Cantilever beams are widely used in atomic force microscopy. The deflection of the cantilever is monitored to measure the atomic forces. Noise analysis of the cantilever beam is especially important for AFM where the ultimate resolution of the system is determined by the thermal mechanical noise of the cantilever.

Consider the cantilever beam depicted in Fig. 3. In this figure, the cantilever is meshed into $5 \mu \mathrm{m}$ by $5 \mu \mathrm{m}$ fournode finite elements. ${ }^{15}$ The length and the width of the cantilever are 100 and $40 \mu \mathrm{m}$, respectively. Hence, the finite

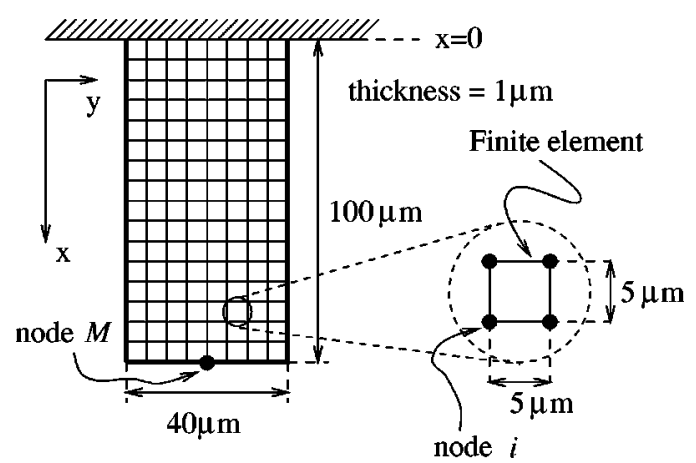

FIG. 3. FEM modeling of a cantilever beam and the electrical model. The length and the width of the cantilever is 100 and $40 \mu \mathrm{m}$, respectively. The thickness of the cantilever is $1 \mu \mathrm{m}$. Cantilever material is silicon (Young modulus, $E=130 \mathrm{~Pa}$, density, $\rho=2.332 \mathrm{~g} / \mathrm{cm}^{3}$, Poisson ratio, $\sigma=0.278$ ). Node $M$ is in the middle of the free end.

element (FE) model of the cantilever beam is composed of 160 elements and 189 nodes. Each node can be viewed as an electrical port. The electrical analog of this mechanical structure is then a 189-port electrical circuit with a 189 by $189 \mathbf{Z}$ matrix.

We can determine the displacement noise of any node on the cantilever by using Eq. (3.7). To calculate the $u_{i} / F_{i}$ ratio in this equation, a sinusoidal force is applied to the node and the displacement of the same node is calculated through the frequency (harmonic) analysis of finite element method (FEM) software, ANSYS 5.4. ${ }^{16} \mathrm{We}$ applied this procedure to the node in the middle of the free end (node $M$ in Fig. 3) to calculate the displacement noise. The rms noise spectrum of node $M$ is depicted in Fig. 4. Two resonance peaks are visible between $100 \mathrm{~Hz}$ and $1 \mathrm{MHz}$. These resonance peaks have nonzero bandwidths, since a small amount of loss is present in the system. The loss coefficient is chosen such that the quality factor of the first resonance is 100 . Without the loss, the quality factor will be infinity and the imaginary part of the displacement phasor, and hence Eq. (3.7) will be zero. The main sources of noise in the cantilever are the structure

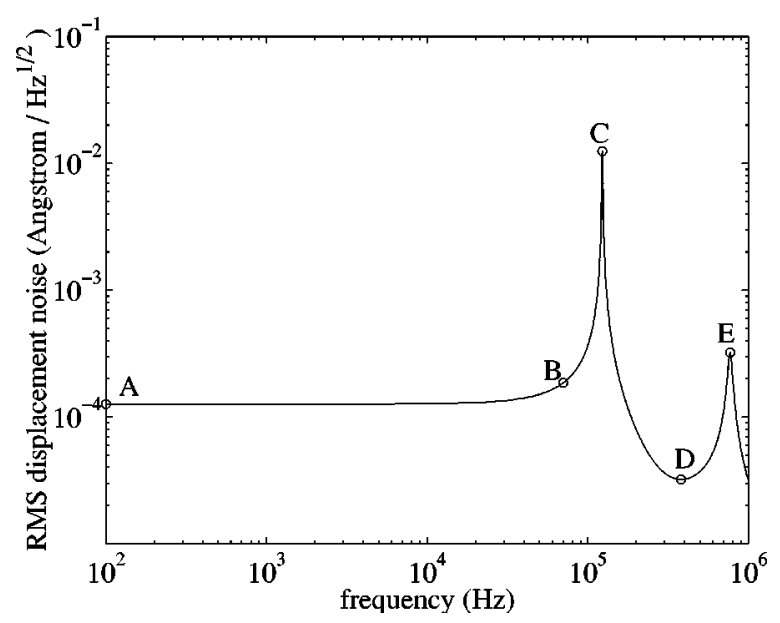

FIG. 4. Calculated rms mechanical noise amplitude $\left(\sqrt{z_{n}^{2}}\right)$ of the free end (node $M$ ) of the cantilever by using FEM and Eq. (3.7) (temperature, $T$, is $300 \mathrm{~K})\left(f_{A}=100 \mathrm{~Hz}, f_{B}=70 \mathrm{kHz}, f_{C}=123 \mathrm{kHz}, f_{D}=380 \mathrm{kHz}, f_{E}=768\right.$ $\mathrm{kHz})$. 


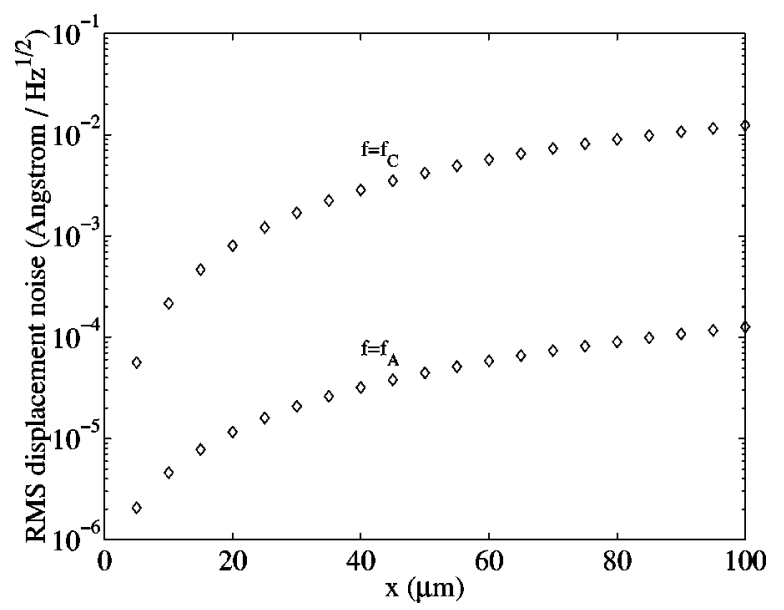

FIG. 5. Calculated rms mechanical noise amplitude $\left(\sqrt{z_{n}^{2}}\right)$ along the cantilever axis at $f=f_{A}=100 \mathrm{~Hz}$ and $f=f_{C}=123 \mathrm{kHz}$. The symbol $\diamond$ shows FE nodes, $i$ (nodes along the cantilever axis).

damping (Rayleigh damping), air damping and coupling to the bulk waves in the cantilever stand.

Figure 5 shows the mechanical noise amplitude variation along the longitudinal axis of the cantilever for two different frequencies $\left(f_{A}\right.$ and $\left.f_{C}\right)$. At each FE node a sinusoidal force is applied and the displacement of the node is calculated by FEM. The rms mechanical noise amplitude is evaluated by using Eq. (3.7). For the cantilever depicted in Fig. 3, noise correlation matrix (N) is 189 by 189 and in Fig. 5 only some of the diagonal elements which correspond to nodes on the cantilever axis are plotted. For the cantilever beam, the noise amplitude increases towards the free end. It is zero at the node $(x=0)$ where the cantilever is fixed. The free end has the most noise. The ratio of the noise amplitude at the resonance to the noise amplitude at very low frequency is around 100 , which is equal to the quality factor of the cantilever.

Correlation of noise between two nodes can be calculated by using FEM and Eq. (3.8). We will use the correlation coefficient to compare noise correlation within the cantilever beam. Correlation coefficient is a unitless variable and defined as

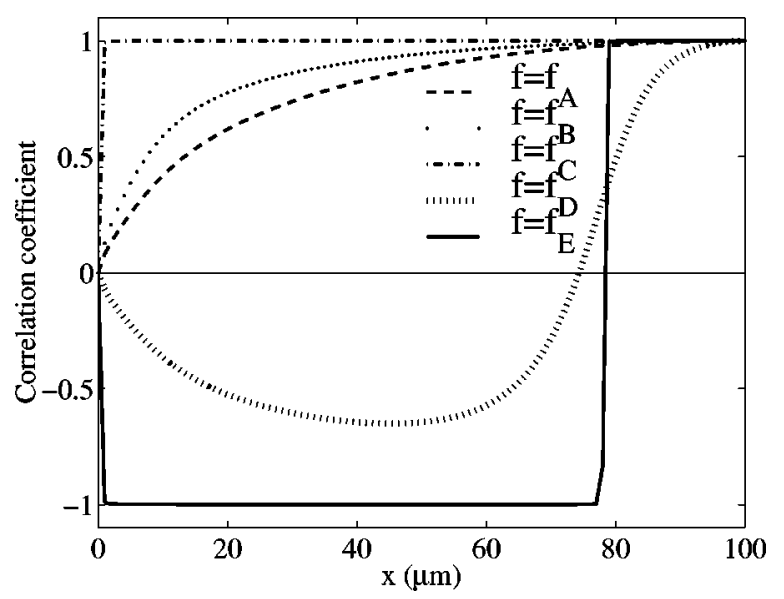

FIG. 6. Correlation coefficient between the nodes along the cantilever axis and the node at the middle of the node $M$.
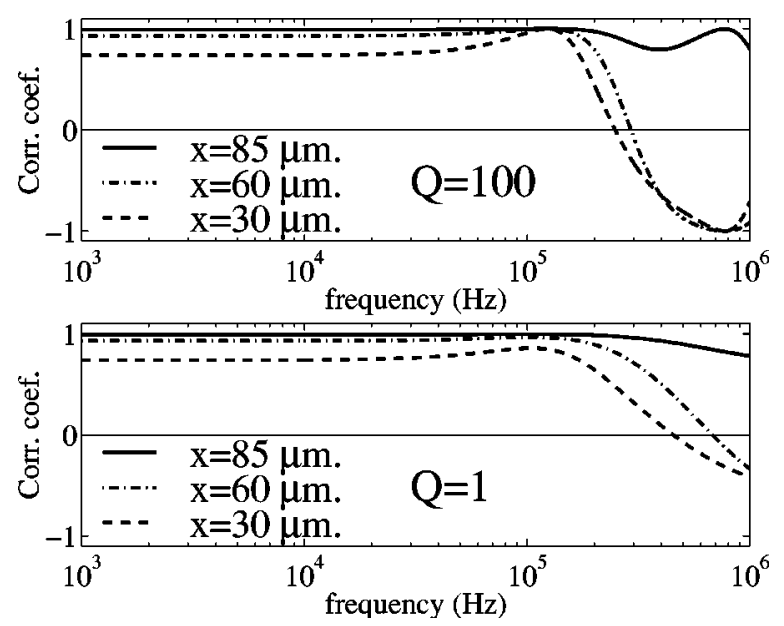

FIG. 7. Correlation coefficient between the node at the free end $(x=100$ $\mu \mathrm{m})$ and the nodes at $x=30 \mu \mathrm{m}, x=60 \mu \mathrm{m}, x=85 \mu \mathrm{m}$ for $Q=100$ and $Q=1$ as a function of frequency.

$$
\rho=\frac{\left\langle u_{n_{i}} u_{n_{k}}^{*}\right\rangle}{\sqrt{\left\langle u_{n_{i}} u_{n_{i}}^{*}\right\rangle\left\langle u_{n_{k}} u_{n_{k}}^{*}\right\rangle}} .
$$

Figure 6 shows the correlation coefficient between the nodes on the cantilever axis and the node $M$ for five different frequencies. We have increased the number of nodes in the FE model so as not to miss any rapid changes of the correlation coefficient. While calculating the numerator of Eq. (4.1), the node $M$ is excited by a sinusoidal force and the displacements of the nodes on the cantilever axis are measured. Figure 6 shows some elements in a row of $\mathbf{N}$. These elements correspond to nodes on the cantilever axis. Below the first resonance frequency, $f=f_{A}$ and $f=f_{B}$, the correlation coefficient is zero at the fixed end, and gradually approaches unity along the axis. When the excitation frequency is equal to the resonance frequency $\left(f=f_{C}\right)$, the correlation coefficient is unity for most of the nodes. On the first resonance the noise of node $M$ is fully correlated with the noise of all the nodes on the cantilever except with those very close to the fixed end. Note that, unity correlation coefficient does not mean that absolute noise values are the same.

Between the first and the second resonance frequencies $\left(f=f_{D}\right)$ the correlation coefficient takes both negative and positive values and it has another zero at $x=75 \mu \mathrm{m}$ which is the node at rest when the cantilever is excited at $f=f_{D}$. At the second resonance frequency $\left(f=f_{E}\right)$, the correlation co-

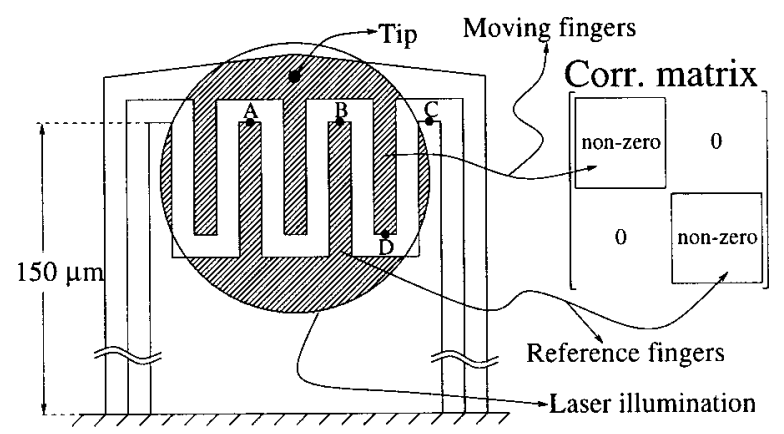

FIG. 8. Interdigital cantilever and the form of correlation matrix. 


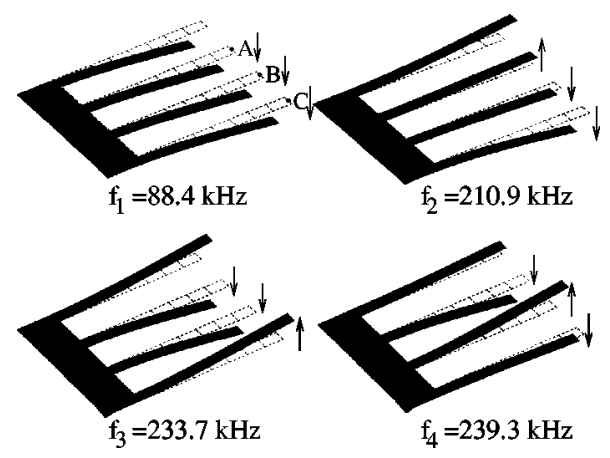

FIG. 9. Mode shapes of the fingers at different resonance frequencies. The length of the reference part is $150 \mu \mathrm{m}$. Finger length is $70 \mu \mathrm{m}$. Arrows indicate the direction of motion. Dotted lines show the undeformed shape of the ID cantilever.

efficient is -1 between the fixed end and the node at rest $(x=78 \mu \mathrm{m})$, which means that this part of the axis is anticorrelated with the free end, whereas other nodes (between the node $M$ and the node at rest) are fully correlated with the free end.

The correlation coefficient can also be calculated as a function of frequency. Figure 7 shows the correlation coefficients between the three different points on the axis and the node $M$ for two different quality factors. For high $Q$ systems, the correlation coefficient is very weakly dependent on $Q$. Only when the quality factor is very low is a significant change observed. We note that, below the first resonance, the correlation coefficient is almost independent of loss.

Interdigital (ID) cantilevers $^{7,8}$ are new interferometric deflection detection sensors for atomic force microscopy. An ID cantilever is composed of two sets of interleaving fingers (reference fingers, moving fingers) to form an optical diffraction grating as depicted in Fig. 8. Such gratings when illuminated by a laser beam reflect incidence light into many orders. The intensities of the orders depend on the relative displacement of the fingers. If a photodetector is used to detect the order intensity, the output current from the detector gives the cantilever deflection. The total noise at the detector output is determined by integrating the mechanical noise within the illuminated area on the cantilever. For the accurate evaluation of the output noise, the noise correlation between fingers should be calculated.

For the ID cantilever, the correlation matrix has the form depicted in Fig. 8 if the FEM nodes are arranged properly. Off-diagonal sub-matrices are zero since there is no mechanical coupling between alternate fingers; for example, the correlation between nodes A (on reference fingers) and D (on moving fingers) is zero. In this section, we will present the correlation coefficient calculation between two reference fingers, specifically between nodes A, B and C as depicted in Fig. 8.

There is a close relationship between resonance mode shapes and the correlation coefficient. Figure 9 shows the mode shapes of the ID cantilever at the first longitudinal resonances. There are four longitudinal resonances between 0 and $300 \mathrm{kHz}$. The length of the fingers is $70 \mu \mathrm{m}$. The cantilever material is silicon and the thickness is $1 \mu \mathrm{m}$. Figure 10 shows the correlation coefficient between nodes A

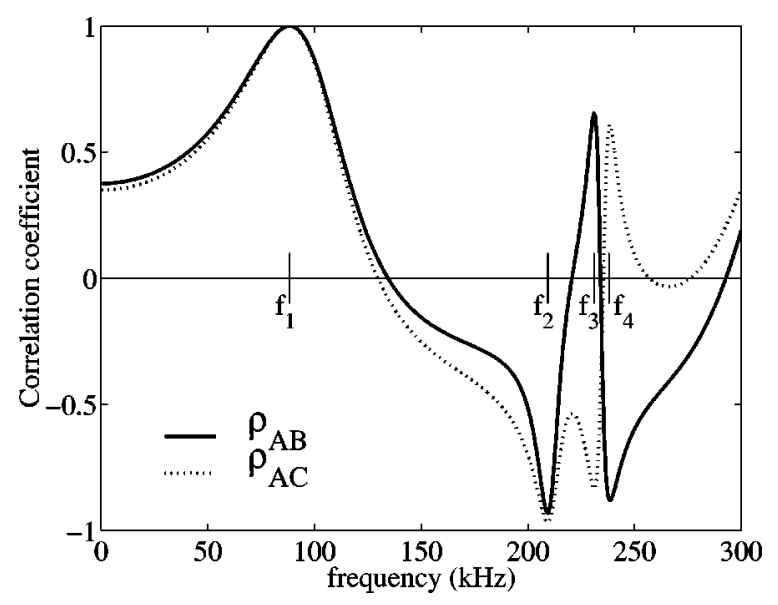

FIG. 10. Calculated correlation coefficients between nodes A and B and between nodes $\mathrm{A}$ and $\mathrm{C}$.

and $\mathrm{B}$ and between nodes $\mathrm{A}$ and $\mathrm{C}$. The resonances reveal themselves as peaks in Fig. 10. At $f_{1}$ all fingers move up and down at the same time as depicted in Fig. 9, hence at this frequency, noise displacements at nodes A, B and C are positively correlated. At $f_{2}, \mathrm{~B}$ and $\mathrm{C}$ move in the opposite direction of A. Hence correlation coefficients, $\rho_{\mathrm{AB}}, \rho_{\mathrm{AC}}$, are negative. At $f_{3}, \mathrm{~A}$ and $\mathrm{B}$ move in the same direction which gives positive correlation, whereas the motions of $\mathrm{A}$ and $\mathrm{C}$ are in the opposite direction as depicted in Fig. 9, hence $\rho_{\mathrm{AC}}$ is negative. At $f_{4}$, the situation is reversed; $\rho_{\mathrm{AB}}$ is negative and $\rho_{\mathrm{AC}}$ is positive.

\section{ACKNOWLEDGMENTS}

The authors would like to thank Professor B. A. Auld and Dr. E. Gustafson of Stanford University for valuable discussions.

${ }^{1}$ Y. Martin, C. C. Williams, and H. K. Wickramasinghe, J. Appl. Phys. 61, 4723 (1987).

${ }^{2}$ N. Osakabe, K. Harada, M. I. Lutwyche, H. Kasai, and A. Tonomura, Appl. Phys. Lett. 70, 940 (1997).

${ }^{3}$ A. Garcia-Valenzue, J. Appl. Phys. 82, 985 (1997).

${ }^{4}$ A. Garcia-Valenzue and J. Villatoro, J. Appl. Phys. 84, 58 (1988).

${ }^{5}$ M. V. Salapaka, H. S. Bergh, J. Lai, A. Majumdar, and E. McFarland, J. Appl. Phys. 81, 2480 (1997).

${ }^{6}$ K. Huang, Statistical Mechanics (Wiley, New York, 1987).

${ }^{7}$ S. R. Manalis, S. C. Minne, A. Atalar, and C. F. Quate, Appl. Phys. Lett. 69, 3944 (1996).

${ }^{8}$ G. G. Yaralioglu, A. Atalar, S. R. Manalis, and C. F. Quate, J. Appl. Phys. 83, 7405 (1998).

${ }^{9}$ P. R. Saulson, Phys. Rev. D 42, 2437 (1990).

${ }^{10}$ H. B. Callen and T. A. Welton, Phys. Rev. 83, 34 (1951).

${ }^{11}$ H. Nyquist, Phys. Rev. 32, 110 (1928).

${ }^{12}$ R. Q. Twiss, J. Appl. Phys. 26, 599 (1955).

${ }^{13}$ S. Seely, Dynamic Systems Analysis (Reinhold, New York, 1964).

${ }^{14}$ N. Nakagawa, B. A. Auld, E. Gustafson, and M. M. Fejer, Rev. Sci. Instrum. 68, 3553 (1997).

${ }^{15}$ A four-node elastic shell element (SHELL63) of ANSYS is used to construct the finite element model for the cantilever.

${ }^{16}$ ANSYS, Inc., 201 Johnson Road, Houston, PA 15342-0065. 